\title{
High normal TSH - risk factor for subclinical hypothyroidism in
}

\section{GH treatment for pituitary dwarfism?}

Alina Daniela Belceanu' ${ }^{1}$, Anamaria Bursuc ${ }^{1}$, Ioana Armaşu ${ }^{1}$, Iulia Crumpei ${ }^{1}$, Georgiana Constantinescu ${ }^{1}$,

Cristina Preda ${ }^{1}$, Mihaela Anton ${ }^{2}$, Carmen Vulpoi ${ }^{1}$

1 - Department of Endocrinology, University of Medicine and Pharmacy "Gr.T. Popa” Iaşi, 2- Department of Endocrinology Bacău

\section{Introduction}

\section{Subclinical hypothyroidism}

$>$ Diagnosed when peripheral thyroid hormone levels are within the normal range, but thyroid stimulating hormone (TSH) is mildly elevated.

$>$ It is common: occurring in $3-8 \%$ of the population, and carries a risk of progression to overt hypothyroidism of 2$5 \%$ per year (1).

$>$ There is no absolute consensus on which patients to treat, although there are some clear recommendations (1).

$>$ The therapeutic benefit of growth hormone (GH) therapy in improving height in short children is widely recognized; however, GH therapy is also associated with other metabolic actions (2).

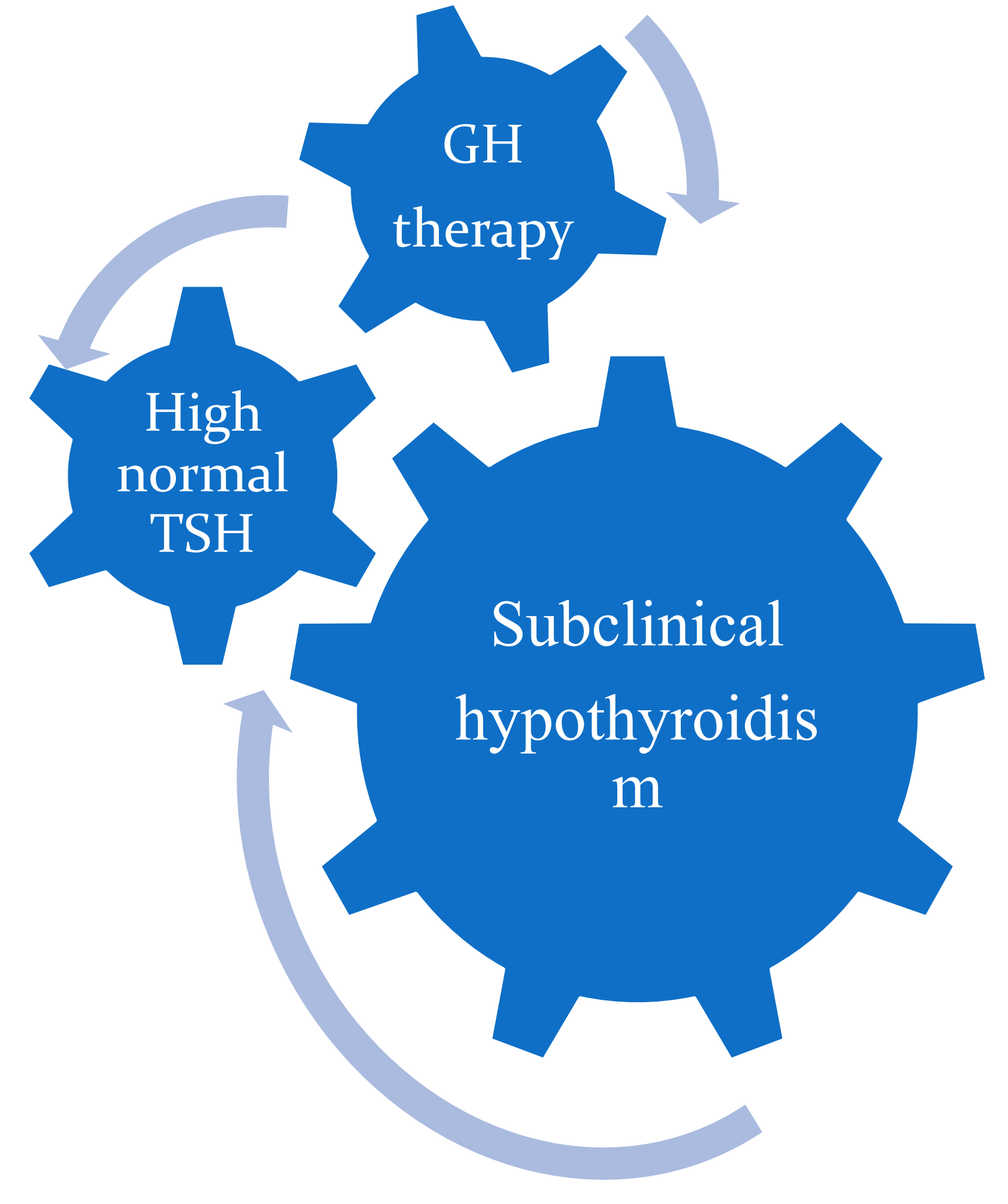

$>$ Normal thyroid hormone secretion or appropriate Lthyroxine substitution is necessary for the optimal effect of recombinant growth hormone (rhGH) on growth rate (3).

$>$ GH therapy in children with GH deficiency (GHD) has yielded conflicting results concerning its impact on thyroid function.

$>$ The decrease of free thyroxine (FT4) levels at recombinant human $\mathrm{GH}(\mathrm{rhGH})$ therapy onset has been reported in several studies (3).

$>$ Data about patients developing subclinical hypothyroidism (SH) are scanty, but it is thought to be associated with impairment of metabolic profile and lower growth response $(4,5)$.

\section{Methods and Results}

\section{Objective:}

To evaluate the effect of rhGH administration on TSH tertiles and $\mathrm{FT}_{4}$ serum concentrations, the frequency of $\mathrm{SH}$ in children with GHD during the 1 st year of treatment, as well as to assess its influence on rhGH therapy effectiveness.

$>$ Retrospective study:

$>$ We reviewed the cases of 75 children with $\mathrm{GH}$ deficiency evaluated at the Endocrinology Department Iasi during one year.

> 59 boys, 16 girls, aged between 4 and 14 years;

$>78,66 \%$ male, $21.34 . \%$ female (Figure 1)

Figure 1: Distribution by gender

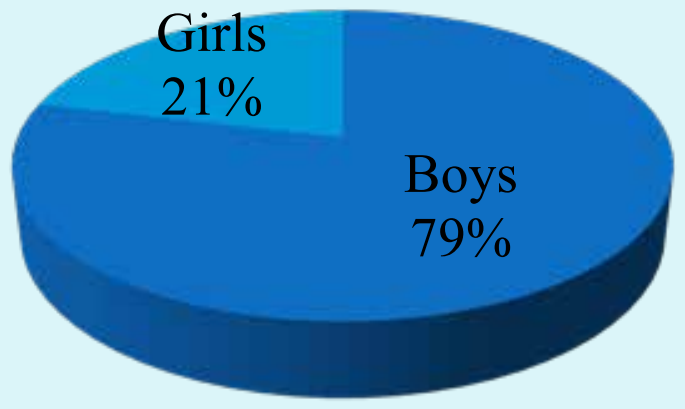

$>$ mean age 8.2 years

$>$ we excluded children treated for genetic syndromes (Turner, Noonan).

$>$ received $0.04+/-0.005 \mathrm{mg} / \mathrm{kg} /$ day $\mathrm{rhGH}$.

At therapy onset all patients :

$>$ had the height below $-2.5 \mathrm{SD}$ (mean SD of -3.2) (Figure 2);

$>$ delayed bone age

$>$ decreased or low normal IGF1 (Figure 3)

$>$ normal thyroid function

After 1 year of rhGH therapy

$>$ Height velocity (HV) improvement was significantly lower in those children who were hypothyroid even for a relatively short period of time during the initial phase of rhGH therapy.

> L-T4 substitution was administered as soon as possible when hypothyroidism was diagnosed (Figure 4).

$>$ To identify children at low, moderate, and high risk for SH we used TSH tertiles.

\section{Discussions}

$>$ The phenomenon of FT4 concentration decrease after rhGH administration in GH-deficient subjects has been reported in several studies $(5,6)$. Moreover, it has been suggested that rhGH therapy might disclose previously unrecognised thyroid insufficiency rather than induce hypothyroidism $(7,8)$. Our observations confirm the phenomenon of „unmasking” central hypothyroidism after rhGH therapy administration in some of children with previous diagnosis of isolated GHD.

$\rightarrow$ Most researchers reported either a lack of significant changes in TSH secretion or a decrease of TSH level in terms of rhGH administration (5,7). This phenomenon has been explained by an increase of somatostatin (being a natural TSH inhibitor) in the patients on rhGH therapy (9).

Figure 3: IGF-1 values
Methods and results:

Clinical and hormonal data (IGF1, TSH, fT $_{4}$ ) as well as radiographic bone assessments were documented at the beginning, 6 months and after first year of rhGH treatment.

Morphological parameters:
Height $(\mathrm{cm})$
Weight $(\mathrm{kg})$
Hheight standard deviation-
SDS
Body mass index-BMI
growth velocity
bone age
progression of puberty

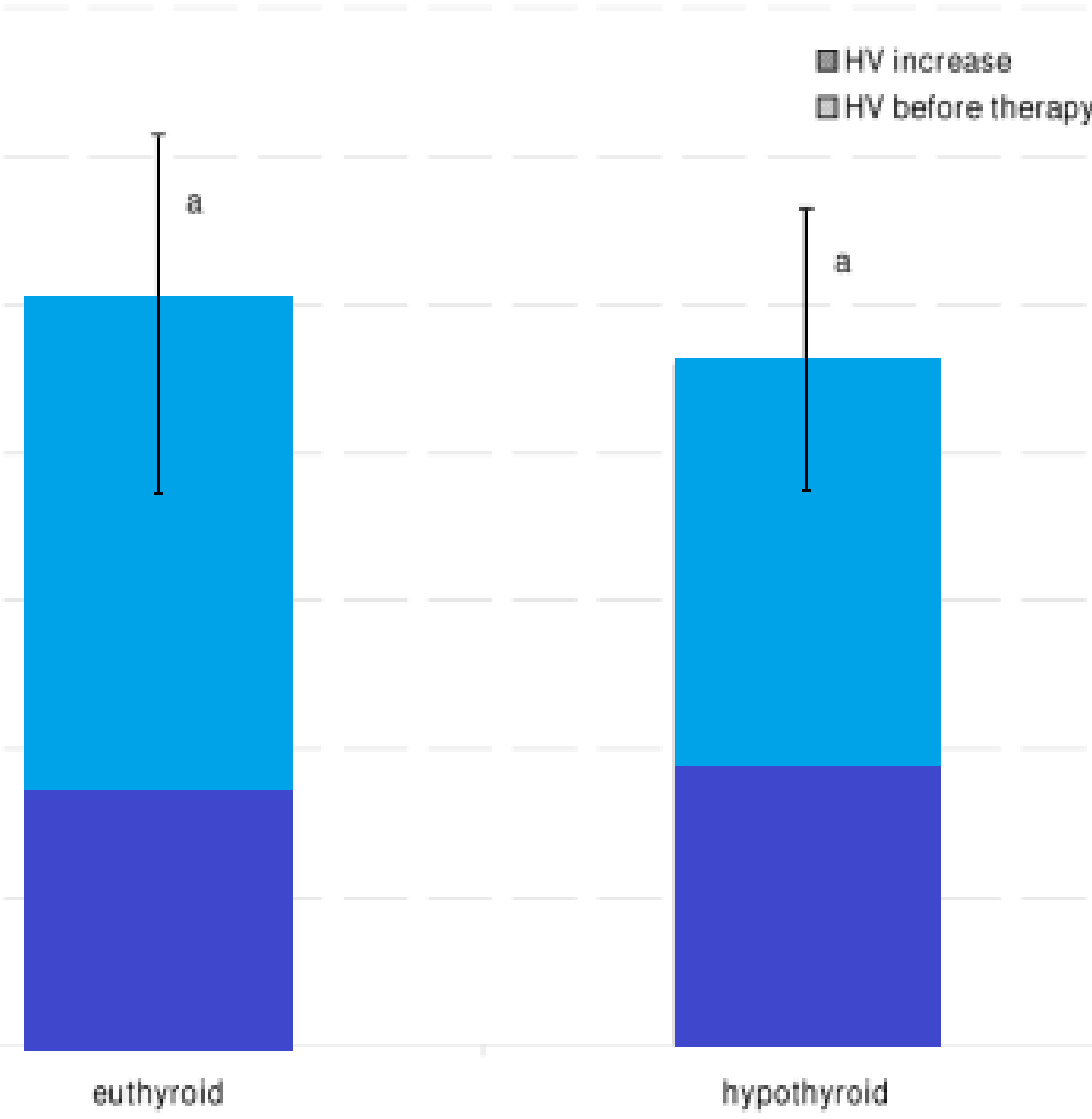

Results:

$>$ All children had an improvement of growth velocity

- Serum IGF-I levels and height SDS significantly increased after 12 months of GH therapy

After one year of rhGH therapy, SH was the only impairment in thyroid function and it was diagnosed in 12 patients $(16 \%)$.

$>$ The risk of SH was increased among subjects with the highest tertile TSH level as compared with subjects with the lowest tertile $(\mathrm{p}<0.05)$.

$>$ Despite similar IGF1 secretion increase, the improvement of height velocity was significantly lower in children with $\mathrm{SH}(0.65$ $\mathrm{cm} /$ month) than in those who remained euthyroid $(0.88$ $\mathrm{cm} /$ month, $P<0.05$ ).

An increase in IGF1 levels was associated with increasing levels of TSH in SH patients and led in four cases to administration of $\mathrm{L}-\mathrm{T}_{4}$ substitution
Figure 4: Height velocity increase in the 1st year of rhGH therapy in the patients divided according to the thyroid function during the initial phase of treatment.

\section{Conclusions}

$>$ The incidence of subclinical hypothyroidism during the first year of rhGH treatment in children with GHD and the influence on the growth rate should be taken into account, as it may worsen the growth response with potential indications to thyroid hormone supplementation.

Our findings suggest that suboptimal thyroid function (highest tertile TSH level) increases vulnerability to the occurrence of SH in children treated with rhGH, needing a closer monitoring. 